\title{
Awareness on Neonatal Care Among Rural Mothers of Reproductive Age Group Admitted in a Tertiary Level Hospital at Mymensingh
}

\author{
*S Huq ${ }^{1}$, SMT Haque ${ }^{2}$, MA Rahman ${ }^{3}$, F Mahmuda $^{4}$, M Mahjabin 5
}

\begin{abstract}
Background: In Bangladesh the neonatal mortality rate is unacceptably high, at 48.4 per 1,000 live births and it is higher in rural areas while the neonatal mortality rate in Asia is 41 per 1,000 live-births, compared to 7 per 1,000 live-births in developed countries.

Materials \& Methods: This descriptive type of cross sectional study was carried out among 180 rural mothers of reproductive age in Mymensingh Medical College Hospital, Mymensingh from October 2013 to January 2014. Data were collected on a pretested questionnaire by face to face interview.

Results: The study revealed that $50.6 \%$ of the respondents were housewife \& education wise $42.2 \%$ had primary education with mean age $26.28 \pm 5.50$ years. $61 \%$ respondents knew that cord should be cut by sterile blade and $65 \%$ respondents mentioned that cord should be cut immediate after delivery. Among them $44.4 \%$ knew about initiation of breast feeding within 1st hour of delivery, $71.1 \%$ believed the importance of colostrums feeding. Only $30 \%$ of the respondents knew about frequency of breast feeding to the baby should be 8-10 times in a day and 58\% had knowledge on exclusive breast feeding. Of the respondents $52.2 \%$ went for exclusive breast feeding but others did not. $67 \%$ respondents immunized their baby whereas $33 \%$ did not. More than half of the respondents (59\%) cleaned their breast before feeding and $80.6 \%$ respondents washed their hands before handling their newborn. A significant association was found between education and knowledge on immunization of newborn $(\mathrm{P}=0.000)$, education and exclusive breast feeding $(\mathrm{P}=0.003)$.

Conclusion: The current study may boost up the awareness among rural mothers of reproductive age group on neonatal care which will further encourage them to be educated and thus neonatal morbidity \& mortality may decline in some extent.
\end{abstract}

Key Words: Neonatal mortality and morbidity rate, Reproductive age, Neonatal care

\section{Introduction}

A newborn infant, or neonate, is a child under 28 days of age. During these first 28 days of life, the child is at highest risk of dying. ${ }^{1}$ During the first 30 days, most of the birth (congenital) defects are discovered. ${ }^{2}$ When a baby enters the world, many body systems change dramatically from the way they functioned during fetal life. So babies need an essential care in neonatal period. ${ }^{3}$ Human infants have a primal need to be carried close to their mother's body. ${ }^{4}$
As early as the $17^{\text {th }}$ and $18^{\text {th }}$ centuries, there were scholarly papers published that attempted to share knowledge of interventions. It was not until 1922, however, that hospitals started grouping the newborn infants into one area, now called the Neonatal Intensive Care Unit (NICU). One in nine babies born in the UK will spend at least a few days in a neonatal unit which specializes in looking after preterm, small and sick babies. ${ }^{5}$

1Dr. Sazia Huq, Lecturer, Department of Public Health, Northern University of Bangladesh

2Dr. Syed Mohammad Tanjilul Haque, Assistant Professor, Department of Forensic Medicine, Anwer Khan Modern Medical College

${ }^{3}$ Dr. Md. Atiqur Rahman, Associate Professor, Department of Community Medicine, Anwer Khan Modern Medical College

${ }^{4}$ Dr. Farzana Mahmuda, Assistant Professor, Department of Pharmacology, Anwer Khan Modern Medical College

5 Maeda Mahjabein, Student of Public Health, Northern University of Bangladesh

*Corresponding Author

Date of submission: 21.11.2017, Date of acceptance: 23.12.2017

AKMMC J 2018; 9(1) : 50-56 
Preterm birth, intra partum-related complications (birth asphyxia or lack of breathing at birth), and infections cause most neonatal deaths. Malnutrition is the underlying contributing factor in about $45 \%$ of all child deaths, making children more vulnerable to severe diseases. ${ }^{6}$ The first week of life is the most critical time for a newborn; three in four newborn deaths occur within the first week, almost 50 percent of them within 24 hours. ${ }^{7}$ An estimated 3.7 million neonatal deaths occur worldwide every year, $98 \%$ of which are in developing countries. In Bangladesh, the neonatal mortality rate (NMR) declined from 63 per 1000 live births in 1985-89 to 34 per 1000 in 2002-06. ${ }^{8}$ Improving the health and nutrition of mothers with providing quality reproductive health services are pivotal to addressing many underlying causes of child mortality. ${ }^{9}$

In Bangladesh, the capital alone has 3.4 million people living in the slums, where the maternal health status is very poor. Although women living proximity of facilities with skilled medical care, $70 \%$ of them in the urban slums give birth at home with untrained traditional birth attendants. ${ }^{10}$ Whereas in Nepal most of the deliveries $(91.5 \%)$ took place at home which were mostly conducted by relatives $(29.6 \%)$ or family members $(35.2 \%)$ or self $(8.5 \%)$. Nearly two third of the respondents $(64.8 \%)$ did not use clean home delivery kit (CHDK) and more than one fourth $(26.8 \%)$ used "blade" for cord cutting. ${ }^{11}$ In Bangladesh, the peri natal (stillbirths and early neonatal) and late neonatal mortality scenario reflects a dreadful picture. ${ }^{12}$ Ninety nine percent (99\%), of maternal and newborn mortality occurs in the developing world, where more than $50 \%$ of women still deliver without the assistance of skilled health personnel. This is a powerful statement about inequity and access to quality care. ${ }^{13}$ Few studies have looked into neonatal morbidity in the community in Bangladesh, and apart from maternal tetanus immunization there are virtually no other programs directed specifically for the neonates. ${ }^{14}$

Neonatal morbidity and mortality rates in Ethiopia are among the highest in the world and stem from a range of socio-economic, political and demographic factors. Many of these deaths are preventable. Around 120,000 newborns die every year and the neonatal mortality rate is 37 per 1000 live births. ${ }^{15}$ Globally, over one million newborn infants could be saved each year by initiating breastfeeding within the first hour of life. In many parts of the world, the rates of early initiation of breastfeeding are extremely low: 17\% in Eastern Europe and Central Asian countries, and $33 \%$ in Asia-Pacific. The highest rates (about 50\%) are in Latin America, the Caribbean, East and North Africa. ${ }^{16}$ The World Health Organization guidelines from 1998 recommend that postnatal care for all newborns should include immediate and exclusive breastfeeding, warming of the infant, hygienic care of the umbilical cord, and timely identification of danger signs with referral and treatment ${ }^{17}$. Countries worldwide are striving towards achieving Millennium Development Goal 4, which deals with the reduction of neonatal deaths. Provision of immediate newborn care and postnatal care are essential in promoting neonatal health. ${ }^{18}$ Malawi is making efforts to reduce intra partum related deaths as a way of achieving Millennium Development Goal 4. In Malawi, the prenatal mortality rate is estimated at 40 deaths per 1,000 births and the neonatal mortality rate is 31 per 1,000 live births, with $71 \%$ skilled attendance for deliveries. ${ }^{19}$ SouthAsian and sub-Saharan African regions account for two thirds of the global burden of neonatal deaths annually. ${ }^{20}$ High infant and maternal mortality rates are one of the biggest health issues in Pakistan. Pakistan's Millennium Development Goals (MDG) aspire to decrease IMR and CMR to 40 and 45 (MDG 4) and MMR to 140 (MDG 5) by 2015. ${ }^{21}$

Universal access to reproductive health and rights was designated as a developmental goal at the 1994 International Conference on Population and Development (ICPD) (UNFPA). This universal access is essential to achieve the millennium development goals for maternal and neonatal health (MDGs 4 and 5) which are to reduce maternal and neonatal mortality to one-third of current levels by 2015. ${ }^{22}$

The present study was aimed to determine the knowledge on neonatal care among rural mothers of reproductive age group admitted in a tertiary level hospital at Mymensingh. 


\section{Methodology}

This descriptive type of cross sectional study was conducted during the period of October 2013 to January 2015 to assess the awareness of neonatal care among the rural mothers of reproductive age admitted in Mymensingh Medical College Hospital of Mymensingh district. Non randomized Purposive sampling technique was applied to select the respondents and face to face interview was taken by using a self-administered and modified questionnaire.

The study was based on primary data with descriptive cross sectional design filled directly with the help of respondents. All the data were entered and analyzed by using Statistical Packages for Social Science (SPSS) software version 16.0, (Chicago, USA). Processed data was presented in the form of percentage, tables, charts and bars. Further, it will analyze significantly with the help of Mean, Standard Deviation, and chi-square test was used to find out the significant association between education and knowledge on immunization of newborn $(\mathrm{P}=0.000)$, education and exclusive breast feeding $(\mathrm{P}=0.003)$. Finally the data was interpreted on the basis of study findings.

\section{Results}

This descriptive type of study cross sectional was conducted among the 180 mothers of reproductive age group from a tertiary level of hospital of Mymensingh. A questionnaire was designed to collect the information. The questionnaire divided into four parts. They are as follows: 1. Sociodemographic characteristics 2. Knowledge related variables 3. Practice related variables 4 . IEC related variables. All data were entered and analyzed by using statistical package for social science (SPSS) software version 16.0 (Chicago).

Among 180 mothers of reproductive age group (1545 years), more than half of the respondents were in the age group 15-25 years. The socio demographic characteristics of the respondents were described in Table-1. The mean age of the study population was $26.28 \pm 5.50$ years. Of the respondents, $61 \%$ knew that during delivery umbilical cord should be cut by sterile blade while $39 \%$ by sterile scissor and more than half of the respondents $(65 \%)$ replied that umbilical cord should be cut immediate after delivery. (Figure-1, 2)

Regarding initiation of breast feeding after delivery only $44.4 \%$ of the respondents knew within $1 \mathrm{st} \mathrm{hr}$ of delivery and others (55.6\%) did not mention about it. Of the respondents $31.1 \%$ opined that bathing of newborn should be done minimum 3 days after birth and others (68.9\%) did not mention appropriately. $71.1 \%$ respondents had knowledge on colostrums feeding and $28.9 \%$ did not know. Among the respondents only $30 \%$ knew about frequency of breast feeding to the baby should be 810 times in a day and $58 \%$ respondents had knowledge on exclusive breast feeding and a significant association was found between education and knowledge on exclusive breast feeding, $(\mathrm{P}=0,003)$. (Table-2)

It was also found from the study that $59 \%$ of the respondents clean their breast before feeding to their baby whereas $41 \%$ did not clean and majority of the respondents $(80.6 \%)$ washed their hands before handling their baby. Among the respondents $61.7 \%$ feed colostrums to their baby and $38.3 \%$ did not. Another two key findings were $52.2 \%$ respondents had practiced exclusive breast feeding while rest (47.8\%) did not practice it and 67\% immunized their baby after birth whereas $33 \%$ did not. (Table-3)

Table-1: Distribution of respondents by Sociodemographic characteristics. $n=180$

\begin{tabular}{|c|c|}
\hline Attribute & Findings \\
\hline Age & $\begin{array}{l}15-25: 56 \%, 26-35: 40 \%, 36-45: 4 \% ; \text { Mean age } \\
( \pm S D)=26.28 \pm 5.50\end{array}$ \\
\hline Education & $\begin{array}{l}\text { Illiterate: } 20 \% \text {, Primary: } 40.2 \% \text {, } \\
\text { Secondary: } 13.3 \% \text {, Higher secondary: } 9.4 \% \text {, } \\
\text { Graduation: } 15 \%\end{array}$ \\
\hline Occupation & $\begin{array}{l}\text { Government job: } 14.4 \% \text {, Private job:20\%, } \\
\text { Business:6.7\%, House wife:50.6\%, Day } \\
\text { labour: } 8.3 \%\end{array}$ \\
\hline Monthly Income(TK) & $\begin{array}{l}\text { 5000-15000:91.6\%, 15001-25000:7.8\%, } \\
\text { 25001-35000:0.6\% }\end{array}$ \\
\hline Number of children & $1: 55 \%, 2: 31 \%, 3: 9.4 \%, 4: 4 \%,>4: 0.6 \%$ \\
\hline
\end{tabular}


Awareness on Neonatal Care Among Rural Mothers of Reproductive

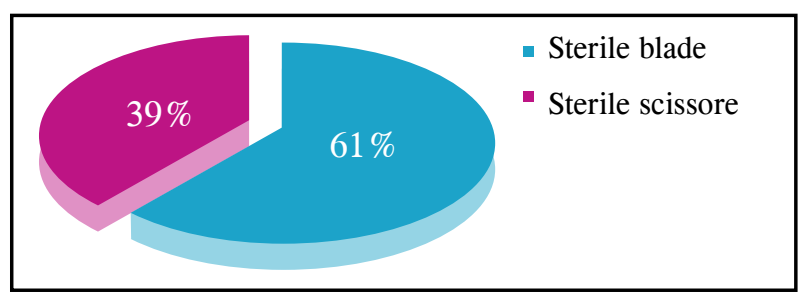

Figure-1: Distribution of respondents by knowledge on type of cord cutting instruments.

It is found from the figure- 1 that $61 \%$ of the respondents knew that cord should be cut by sterile blade \& $39 \%$ by sterile scissor.

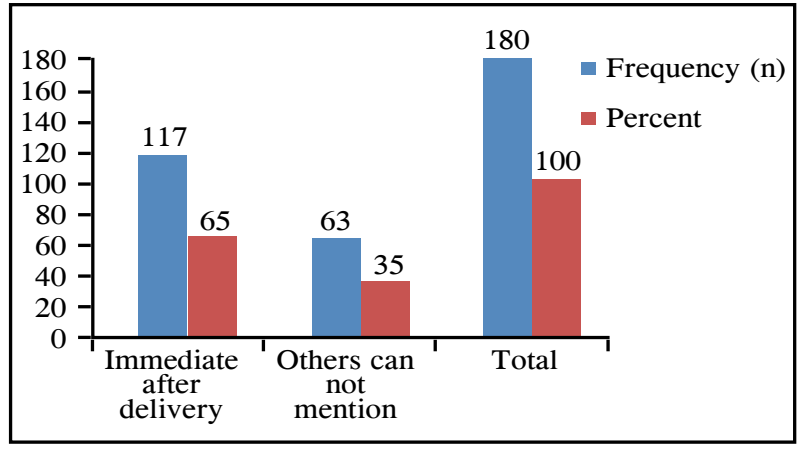

Figure-2: Distribution of the respondents by knowledge on time of cord cutting after delivery

Figure-2 shows that more than half of the respondents $(65 \%)$ replied that umbilical cord should be cut immediate after delivery and others $35 \%$ could not mention accurately.

Table-2: Distribution of the respondents by association between education and knowledge on exclusive breast feeding

\begin{tabular}{ccccccccc}
\hline $\begin{array}{c}\text { knowledge } \\
\text { on exclusive } \\
\text { breast } \\
\text { feeding }\end{array}$ & $\begin{array}{c}\text { Illite- } \\
\text { rate }\end{array}$ & $\begin{array}{c}\text { Pri- } \\
\text { mary }\end{array}$ & $\begin{array}{c}\text { Secon- } \\
\text { dary }\end{array}$ & $\begin{array}{c}\text { Higher } \\
\text { secondary }\end{array}$ & $\begin{array}{c}\text { Graduation } \\
\text { and above }\end{array}$ & Total & $\begin{array}{c}\text { P- } \\
\text { Value }\end{array}$ \\
\hline Yes & 4 & 46 & 19 & 10 & 26 & 105 & \\
No & 32 & 30 & 5 & 7 & 1 & 75 & .000 \\
Total & 36 & 76 & 24 & 17 & 27 & 180 & \\
\hline
\end{tabular}

Table-3: Distribution of the respondents by practice related variables. $(n=180)$

\begin{tabular}{ll}
\hline Practices & Findings \\
\hline Cleaning of breast before feeding & Yes:59\%, No:41\% \\
Washing hand before handling newborn & Yes:80.6\%, No:19.4\% \\
Feeding colostrums to newborn & Yes:61.7\%, No:38.3\% \\
Exclusive breast feeding & Yes:52.2\%, No:47.8\% \\
Immunized their baby & Yes:67\%, No:33\% \\
\hline
\end{tabular}

\section{Discussion}

The descriptive type of cross sectional study was conducted to find out the awareness on neonatal care among rural mothers of reproductive age group in Bangladesh and to find out the associated factors related with neonatal care in Bangladesh. It was revealed that most of the respondents $(56 \%)$ were in age group $15-25$ years \& rests $(40 \%$ \& $4.4 \%)$ were in 26-35 \& 36-45 years of age group respectively. It was found from the study $50.6 \%$ of the respondents were housewife while $20 \%, 14.4 \%, 8.3 \%$ and $6.7 \%$ were involved in private job, government job, day labor and business respectively. A study was carried out in a rural community of Baitadi district of Nepal in 2010 and it was seen that most of the respondents $(62.0 \%)$ belonged to the age group 20 to 30 years with the mean age of $24.5 \pm 2.9$ years. Agriculture was the major occupation (91.5\%) of the mothers ${ }^{23}$. These findings showed that this study were closer to the current study in age, but different in occupation due to socio-cultural difference between two countries.

Among the respondents $42.2 \%$ were primary passed, $20 \%$ were illiterate, followed by $13.3 \%$, $9.4 \% \& 15 \%$ were secondary, higher secondary passed and completed their graduation. A study done in the Kassena-Nankana District (KND) of the Upper East region of Northern Ghana in the year $2009^{24}$ and it was explored that More than $90 \%$ of mothers had either no formal education or education that ended after primary or junior secondary school. The findings revealed that there were no similarities with the present study. It may be due to difference of social status between two countries.

The study explored that more than half of the respondents $(55 \%)$ had one children, followed by $31 \%$ had two children, $9.4 \%$ had three, $4 \%$ had four children and $0.6 \%$ had more than four children respectively. A study was conducted in the Southern region of Malawi in the Chiradzulu district. ${ }^{19}$ It revealed parity of the participants ranged from one to seven and the majority of the participants had given birth between two to four times. The findings showed that it has some dissimilarity with the presents study may be due to inappropriate knowledge on family planning among the Africans. It was also seen that $61 \%$ of the respondents know that cord should be cut by sterile blade \& $39 \%$ by 
sterile scissor. More than half of the respondents $(65 \%)$ replied that umbilical cord should be cut immediate after delivery and others $35 \%$ can not mention accurately. The study also showed that $44.4 \%$ of the respondents knew that breast milk should be started within 1 st hr of delivery and others $(55.6 \%)$ could not mention it. $31.1 \%$ of the respondents opined that bathing of newborn should be done minimum 3 days after birth and others $(68.9 \%)$ can not mention appropriately. Among the respondents $71.1 \%$ had knowledge on colostrums feeding while $28.9 \%$ did not know and $61.7 \%$ feed colostrums to their baby and $38.3 \%$ did not. Of the respondents $67 \%$ immunized their newborn after birth whereas $33 \%$ did not. A study done in Siddheshwar, Siddhapur and Gujar VDCs of Baitadi districts of Nepal in $2011^{11}$ revealed nearly two third of the respondents $(64.8 \%)$ did not use clean home delivery kit (CHDK) and more than one fourth $(26.8 \%)$ used "used blade" for cord cutting. More than two third (38\%) of the respondents bathed their babies within one hour and only $18.3 \%$ of respondents bathed their babies after 24 hours. Only $7.0 \%$ of the respondents' breast fed their babies within one hour, though all babies were breast fed. Nearly one third $(29.6 \%)$ of the respondents discarded the first milk (colostrums). More than one third of the respondents (35.2\%) did not give vaccine to their babies. There were only one similarity on immunization of baby but rests of the findings of these two studies were dissimilar and this proves the lack of awareness, traditional cultural practices and lack of free time for the mothers in Nepal which is leading Nepal towards a harmful neonatal practice.

A study also examined the prevalence of colostrums feeding and time to initiation of breast-feeding in 143 rural Bangladeshi women in Matlab thana. ${ }^{25}$ It revealed that $99 \%$ of the mothers reported feeding their newborn colostrums. 59\% per cent of mothers initiated breast-feeding within 4 hours, and $88 \%$ within 12 hours of parturition. It showed that there were dissimilarities with the current study may be due to socio-cultural variation.

Among the respondents $30 \%$ of the respondents knew about frequency of breast feeding to the baby should be 8-10 times in a day \& others $70 \%$ respondents could not give correct information. The study also revealed that $58 \%$ respondents had knowledge on exclusive breast feeding whereas $42 \%$ did not and more than half of the (59\%) respondents clean their breast before feeding and $41 \%$ did not clean. Regarding hands washing before handling baby $80.6 \%$ wash their hands and $19.4 \%$ did not wash. Another finding was $52.2 \%$ respondents had practiced exclusive breast feeding and rest $(47.8 \%)$ did not practice it. A cohort study was designed according to breastfeeding status in infants from birth to six months of age. ${ }^{26}$ The data showed that only $38 \%$ of children aged 2-3 months are exclusively breastfed and $23 \%$ of children are given complementary foods before the sixth month. It was seen that the improvement was in exclusive breastfeeding of the mother compared to the present study due to increasing knowledge of the mother.

A significant association was found between education and knowledge on immunization of newborn $(\mathrm{P}=0.000)$, education and exclusive breast feeding $(\mathrm{P}=0.003)$.

\section{Conclusion}

The population of our country is increasing in an alarming rate. The growth rate of our population creates problem in every sphere of our life. Poor neonatal care is a serious public health problem. Still the neonatal \& infant mortality rate is high in Bangladesh. Unless the neonatal care is proper, probability remains of frequent infections in infancy causing in poor brain development and consequently resulting is an increased health burden for the state. The study revealed the gaps in different vital areas for neonatal care like ignorance about exclusive breastfeeding, usefulness of colostrums, initiation of breast feeding, minimum time period for breastfeeding which are not good parameters for proper neonatal care.

Unless neonatal care is proper increases mortality \& morbidity rate will add to the existing public health problem. So this problem should be given adequate attention to address this burning issue of public health of this country. So that people must be made conscious through education.

\section{Recommendations}

- Health education should be strengthened through mass media and health services to educate the mothers for nursing of their neonates. 
- Awareness should be created by effective training program about the impact of good health of children.

- Mothers should be educated about breast feeding and weaning.

- Awareness should be created among mothers for the necessity of timely immunization.

\section{Conflict of interest: None}

\section{References}

1. WHO. Health topics on infants, neonate. 2006 [Serial online] [cited 5 Oct 2013]; Available from: URL:http:/ /www. who.int/ opics/infant_ newborn/en/

2. Medline plus. US. National Library of Medicine. National Institutes of Health. 2011 [serial online] [cited 5 Oct 2013]; Available from:URL:http://www.nlm.nih.gov /medlineplus /ency/article/ 002271.htm

3. The Neonatal Intensive Care Unit. Lucile Packard Children's Hospital. Stanford University School of Medicine. 2013 [serial online] [cited 4 Oct 2013]; Available from: URL:http: // www. lpch.org/ Disease HealthInfo/ Health Library/ hrnewborn/ nicuintr. html

4. "Infants' Need for Touch". Human Development 45(2): 100-103. 2013 [serial online] [cited 13 Sep 2013]; Available from: URL: http://en. wikipedia. org/ wiki/Infant

5. Bliss about Neonatal Care. 2013 [serial online] [cited 4 Oct 2013]; Available from: URL: http://www.bliss.org.uk/help-for-families/inhospital/about-neonatal-care/

6. WHO. Children: Reducing Mortality. 2013 [Serial Online] [cited 6 Oct 2013]; Available from: URL: http://www.who.int/mediacentre/ factsheets/fs178/en/

7. Maternal and Neonatal Health in Bangladesh. Statistics from UNICEF State of the World's Children 2009 report and BDHS 2007. 2009 [serial online] [Cited 6 Oct 2013]; Available from: URL: http:// www.unicef.org/ bangladesh/ Maternal_and_Neonatal_Health.pdf
8. Azad K, Barnett S, Banerjee B, et al; Effect of scaling up women's groups on birth outcomes in three rural districts in Bangladesh: a clusterrandomized controlled trial. Apr 2010; 375(9721): 1193-1202.

9. Warren C, KivunagaJ. Safe Motherhood Community based Survey. Ethiop. J. Health Dev. 2010; 24(1).

10. Nahar S, Banu P, Hashima M, et al. Womenfocused development intervention reduces delays in accessing emergency obstetric care in urban slums in Bangladesh. BMC Pregnancy and Childbirth. 2011; 11: 11.

11. Devkota M, Bhatt M. Newborn Care Practices of Mothers in a Rural Community in Baitadi, Nepal. Nepal Journals Online. Health Prospect. 2011; 10: 5-9.

12. Syed U, Asiruddin S, Helal S, et al. Immediate and Early Postnatal Care for Mothers and Newborns in Rural Bangladesh. Journal of Health, Population and Nutrition. Dec 2006; 24(4): 508-518.

13. Lemovne. Maternal and newborn Health. 2012 [Serial Online] [cited 6 Oct 2013]; Available from: URL:http:// www.unicef. org/ health/ index_maternalhealth.html

14. Ahmed S, Sobhan F, Islam A. Neonatal Morbidity and Care-seeking Behavior in Rural Areas of Bangladesh. ICDDR, B Bangladesh. Mohakhali, Dhaka 1212. 1998 [serial Online] [cited 17 Oct 2013]; Available from: URL: https:// centre.icddrb.org/ images/ wp114.pdf

15. Bhutta Z, Darmstad G, Hasan B, et al. Community-based interventions for improving peri-natal and neonatal health outcomes in developing countries. A review of the evidence. Pediatrics. 2005; 115(2): 519-617.

16. Janna A. Interventions for promoting the initiation of breastfeeding. 2014 [Serial online] [Cited on 20 Mar 2014]; Available from:URL: http://apps.who.int/rhl/pregnancy_childbirth/ca re_after_childbirth/cd001688_JanaAK_ com/en/ 
17. Aminuzzaman $S$, Anwar I, M Sami. Inequity in maternal health-care services. Bulletin of the World Health Organization. 86(4): 8.

18. Kumbani L, Bjune G, Chirwa E, et al. Why some women fail to give birth at health facilities. Reproductive Health 2013; 10: 9

19. Van R, White S, Ntonya C, et al. Reproductive health in rural Malawi: a population-based survey. 2003; 110: 902-908.

20. Kruk M, Mbaruku G, McCord W, et al. Bypassing primary care facilities for childbirth. Health Policy Plan. 2009; 24: 279-28829.

21. Global Action for Children. 2013 [serial online] [cited 9 Oct 2013]; Available from: URL: http:/ /www. apfmj. com/ content/10/1/11.

22. Nafisa L, Tracey $\mathrm{K}$, Jahan A, et al. Communication barriers in maternal and neonatal emergencies in rural Bangladesh. International Journal of Sociology and Anthropology. Oct 2012; 4(8): 226- 237.
23. Devkota M, Bhatta M. Newborn Care Practices of Mothers in a Rural Community in Baitadi. Nepal, Institute of Medicine. Health Prospect; 2011: 10 .

24. Welaga P, Moyer A, Aborigo R, et al. Why Are Babies Dying in the First Month after Birth? A 7-Year Study of Neonatal Mortality in Northern Ghana. 2013 [serial online] [cited 9 0ct 2013]; Available from: URL: http://www. plosone.org/ article/info:doi/10.1371/ journal. pone. 0058924

25. Holman DJ, Grimes MA. Colostrum feeding behaviour and initiation of breast-feeding in rural Bangladesh. US National Library of Medicine National Institutes of Health. 2001 Jan; 33(1): 139-54.

26. Mihrshahi S, Wendy $\mathrm{H}$, Jennifer $\mathrm{K}$, et al. Association between infant feeding patterns and diarrhoeal and respiratory illness: A cohort study in Chittagong, Bangladesh. International breastfeeding journal. 2008; $3: 28$ 